\title{
Phytohaemagglutinin and transfer factor in the leucocyte migration inhibition test in patients with sarcoidosis
}

\author{
W. L. MOR I S O ${ }^{1}$ \\ Department of Dermatology, St. Helier Hospital, Carshalton, Surrey
}

\begin{abstract}
Morison, W. L. (1970. Thorax, 31, 87-90. Phytohaemagglutinin and transfer factor in the leucocyte migration inhibition test in patients with sarcoidosis. A new system of phytohaemagglutinin stimulation in vitro using the leucocyte migration inhibition test has been applied to patients with active and inactive sarcoidosis and subjects with a persistently negative response to tuberculin. Patients with active sarcoidosis and the tuberculin-negative subjects have a depressed response to the mitogen. This response is improved by the addition of transfer factor to the cultures.
\end{abstract}

Phytohaemagglutinin (PHA) stimulation in the lymphocyte transformation test has been used for several years as a non-specific measure of cellmediated immune function. Recently, an alternative method for measuring response to PHA has been described using the leucocyte migration inhibition test system (Morison, 1974).

The present study was undertaken to explore some of the possibilities of this test in the investigation of patients with active and inactive sarcoidosis and subjects with a persistently negative response to tuberculin. In addition, the influence of transfer factor on the results was examined.

\section{MATERIALS AND METHODS}

PATIENTS Three groups were investigated:

10 patients with active sarcoidosis. The diagnosis had been established on clinical, histological, and radiological grounds. There were five men and five women, mean age 40 with a range of $25-56$ years. Two of the patients had received prednisone, $5 \mathrm{mg}$ daily, over the preceding three and six months.

5 patients with inactive sarcoidosis. These patients had all had bilateral hilar lymphadenopathy, a negative Mantoux test, and a positive histological diagnosis established. They had been free of clinical and radiological evidence of disease for at least one year. There were two men and three women in the group, mean age 41 with a range of 34-58 years.

4 subjects with negative Mantoux reactions at

'Present address: Department of Dermatology, Massachusetts General Hospital, Boston, Massachusetts, USA
1:1000. Each of these subjects had received BCG on at least two occasions; all worked in hospitals and two had worked for more than a year in contact with open cases of tuberculosis. There were three women and one man, mean age 29 with a range of 22-40 years. All were fit and healthy, had no history of significant illnesses, were taking no regular medications, and had normal chest radiographs.

CONTROL SUBJECTS Sixteen healthy control subjects were examined, eight men and eight women, mean age 43 with a range of $24-74$ years. All gave positive Mantoux reactions at 1:1000 dilution.

LEUCOCYTE MIGRATION INHIBITION (LMI) TEST The details of this test and its modification for use with PHA are described elsewhere (Morison, 1974). In summary, it consists of separating the leucocytes from a venous blood sample and packing them into capillary tubes which are then mounted in culture chambers containing tissue culture medium and the mitogen. PHA (Wellcome, freeze dried) was used in a concentration of $5 \mu \mathrm{g} / \mathrm{ml}$ for all tests in this study. The chambers are incubated for 18 hours, during which time the cells migrate fanwise out of the tubes. The area of migration is mapped out by projection microscopy and measured by planimetry. Results are expressed as a migration index, which is the ratio of the area of migration in the presence of mitogen to the area of migration in control cultures. Previous work has suggested that normal subjects have a migration index of less than 0.30 at a PHA con- 
centration of $5 \mu \mathrm{g} / \mathrm{ml}$; between $0.30-0.50$ is equivocal and above 0.50 is abnormal.

TRANSFER FACTOR A quantity of transfer factor was prepared from a normally responsive subject according to the method of Lawrence (Lawrence and AlAskari, 1971). In summary, the method is: the leucocytes are separated from $500 \mathrm{ml}$ venous blood, frozen, and thawed 10 times, treated with pancreatic deoxyribonuclease, and then dialysed across a cellophane membrane against normal saline at $4^{\circ} \mathrm{C}$ for 24 hours. The dialysate is then filtered through a millipore filter before use. The amount of transfer factor added to each culture chamber in the present study was the amount extracted from the leucocytes contained in $10 \mathrm{ml}$ of donor blood.

\section{RESULTS}

The results of PHA stimulation are shown in the Figure. It can be seen that the mean migration index

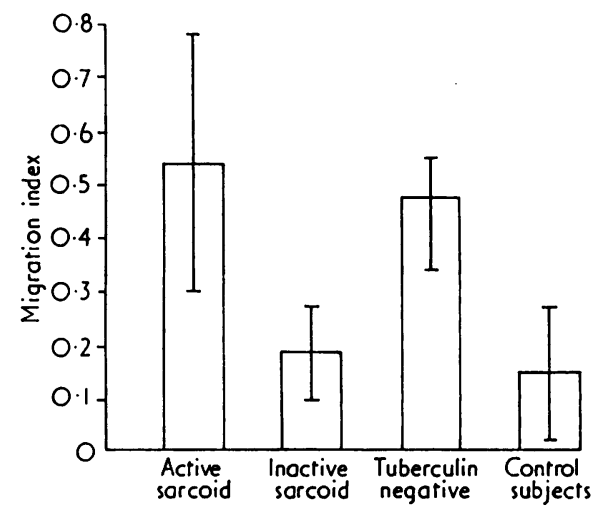

FIG. 1. Mean and range of migration indices on PHA stimulation.

in patients with active sarcoidosis is abnormal (0.54). Six of the 10 patients had a result above 0.50 and all were above 0.30 . In contrast, all patients with inactive sarcoidosis had a result below 0.30 , and the mean index of 0.19 is similar to the mean index of 0.15 in the controls. The tuberculin negative group had a mean index similar to that found in the active sarcoid group and all had an index above 0.30.

The results obtained on the addition of transfer factor to the cultures are shown in the Table. Only seven of the patients with active sarcoidosis were examined and their mean migration index was reduced from 0.52 to 0.23 in the presence of transfer factor. In six of the seven, the index fell below $\mathbf{0 . 3 0}$ while one patient showed no response. The patients with inactive sarcoidosis, like the controls, showed no
T A B L E

\begin{tabular}{|c|c|c|}
\hline & \multicolumn{2}{|c|}{ Mean Migration Index } \\
\hline & PHA & $\begin{array}{c}\text { PHA + } \\
\text { Transfer } \\
\text { Factor }\end{array}$ \\
\hline $\begin{array}{l}\text { Active sarcoid } \\
\text { Inactive sarcoid } \\
\text { Tuberculin negative } \\
\text { Control subjects }\end{array}$ & $\begin{array}{l}0.52 \\
0.19 \\
0.48 \\
0.15\end{array}$ & $\begin{array}{l}0 \cdot 23 \\
0 \cdot 20 \\
0 \cdot 39 \\
0 \cdot 14\end{array}$ \\
\hline
\end{tabular}

significant change. The tuberculin negative group did $\vec{\infty}$ show an alteration, one having a moderate reduction

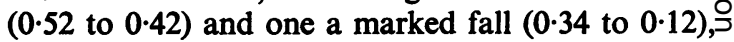
while the other two remained unchanged in the pres- $\overrightarrow{-}$ ence of transfer factor.

\section{DISCUSSION}

Depression of cell-mediated immune function in $\overrightarrow{0}$ some patients with sarcoidosis has been demonstrated both in vivo and in vitro by many investigators. Often the $T$ cell mitogen PHA in the lymphocyte trans-0 formation test has been used as a measure of this depression. While being a useful research tool, the test has several disadvantages which make its wider $\frac{\mathscr{D}}{\mathscr{D}}$ use for routine investigation difficult. It is time- 0 consuming to set up and this limits the number of $\overrightarrow{\overrightarrow{0}}$ patients investigated; a result is not available for ${ }^{3}$ three days or more; and it requires sophisticated skills and equipment. Recently (Morison, 1974), amodification of the leucocyte migration limitation test, using PHA stimulation, has been described.응 The result for this test can be available within $24 \times$ hours, equipment is minimal, and several patients 3 can be examined at the same time. The test can be done in a routine laboratory and therefore permitso the evaluation of cellular immune function in $a_{0}$ greater number of patients with conditions such assarcoidosis.

The present study indicates that response to PHA in this test system is relatively depressed in patients with active sarcoidosis. Using the lymphocyte transformation test, the response to PHA has been variously reported as normal (Girard, Poupon, and ${ }^{\mathrm{c}}$ Press, 1971; Horsmanheimo, 1972) and abnormate (Hirschhorn et al., 1964; Buckley, Nagaya, and을 Sieker, 1966). Recently, it has been suggested (Mangi et al., 1974a) that steroids used for treatment may explain these contradictory results and be at least $-{ }^{-}$ partly responsible for an abnormal result. However, $\overrightarrow{,}$ this does not explain the findings in the present study? as none of the results in patients with active sarcoi- $\stackrel{0}{0}$ dosis was normal and only two of the patients were on a low dose of steroids. 
The response to PHA in patients with inactive sarcoidosis was within normal limits in all cases. This agrees with an in vivo study of cellular immunity using tuberculin reactivity (Selvoos and Niemisto, 1972) in which it was found that although immunity may be depressed during the active phase of sarcoidosis, it returns to normal in most cases with resolution of the disease. In contrast, an in vitro study including PHA stimulation found some impairment of immune function remained after clinical and radiological resolution of sarcoidosis (Osada et al., 1972). A large prospective study on patients known to be tuberculin positive before the sarcoidosis, correlating in vivo and in vitro tests, is required to resolve these conflicting results.

The precise nature of transfer factor is unknown but it is established that dialysable extracts of leucocytes can transfer cellular immunity and activate such immunity when it is depressed. Transfer factor has been shown to have an effect on the depression of cellular immunity in sarcoidosis. An in vivo study (Lawrence and Zweiman, 1968) demonstrated that sensitivity to tuberculin could be transferred locally to the site of injection of transfer factor but systemic spread of this sensitivity in patients with sarcoidosis was very weak. In addition, it has been shown (Valentine and Lawrence, 1969) that transfer factor can, under in vitro conditions, increase the sensitivity of lymphocytes to an added antigen. The present work suggests that this also applies to a mitogen since six of the seven patients with sarcoidosis and two of the tuberculin negative subjects showed an increased sensitivity to PHA in the presence of transfer factor.

The question arises: does this finding lead to a better understanding of the mechanism of the defect in cellular immunity in sarcoidosis? Recent findings suggest that a serum inhibitor is responsible, at least in part, for the defect (Belcher, Carney, and Nankervis, 1974; Mangi et al., 1974b). However, the presence of the inhibitor did not correlate with the cellular immune depression. Furthermore, under the conditions of the LMI test, serum inhibitors are unlikely to be important since the patient's serum is removed by washing. Therefore, although such inhibitors may be partially responsible for the defect, other factors must also be operating. The in vivo study of Lawrence and Zweiman coupled with the present in vitro study suggest that lymphocyte function, impaired response to mediators such as transfer factor, or even a deficiency of such mediators could be playing a part.

The abnormal results in the tuberculin negative subjects were somewhat surprising since apart from their failure to convert with BCG, they had no clinical indication of a more general defect of cellular immunity. The response to transfer factor in two of the subjects is further evidence that immune function in such people is abnormal. No other reports of in vitro studies on a similar group could be found and the present work suggests that further investigation of such subjects is needed.

Dr. H. F. Harwood and Dr. M. Hill kindly consented to their patients being examined and were extremely helpful in co-operating with the study. The work was supported by a grant from the SW Metropolitan Regional Hospital Board. This study was reported in part as an abstract in the Proceedings of the Royal Society of Medicine (1974), 67, 597.

\section{REFERENCES}

Belcher, R. W., Carney, J. F., and Nankervis, G. A. (1974). Effect of sera from patients with sarcoidosis on in vitro lymphocyte response. International Archives of Allergy, 46, 183.

Buckley, C. E., Nagaya, H., and Sieker, H. O. (1966). Altered immunologic activity in sarcoidosis. Annals of Internal Medicine, 64, 508.

Girard, J. P., Poupon, M. F., and Press, P. (1971). Cultures of peripheral blood lymphocytes from sarcoidosis: response to mitogenic factor. International Archives of Allergy, 41, 604.

Hirschhorn, K., Schreibman, R. R., Bach, F. H., and Siltzbach, L. E. (1964). In vitro studies of lymphocytes from patients with sarcoidosis and lymphoproliferative diseases. Lancet, $2,842$.

Horsmanheimo, M. (1972). Phytohaemagglutinin-, tuberculin- and Kveim-induced blast transformation in sarcoidosis. Proceedings of the VI International Conference on Sarcoidosis, edited by K. Iwai and Y. Hosoda, p. 177. University Park Press.

Lawrence, H. S. and Al-Askari, S. (1971). The preparation and purification of transfer factor. In In Vitro Methods in Cell-Mediated Immunity, edited by B. R. Bloom and P. R. Glade, p. 531. Academic Press, New York.

—_ and Zweiman, B. (1968). Transfer factor deficiency response-a mechanism of anergy in Boeck's sarcoidosis. Transactions of the Association of American Physicians, 81, 240.

Mangi, R. J., Dwyer, J. M., Gee, B., and Kantor, F. S. (1974a). The immunological competence of subjects with sarcoidosis. Clinical and Experimental Immuno$\log y, 18,505$.

$\ldots, \ldots, \ldots$, and _- $-(1974 \mathrm{~b})$. The effect of plasma upon lymphocyte response in vitro. Clinical and Experimental Immunology, 18, 519.

Morison, W. L. (1974). Effect of phytohaemagglutinin in the leucocyte migration inhibition test as a measure of cell-mediated immunity. Journal of Clinical Pathology, 27, 113.

Osada, H., Hosoda, Y., Odaka, M., Matsumoto, M., Takahashi, M., Yanaka, M., and Chiba, Y. (1972). Impaired lymphocytes in patients recovered from sarcoidosis. Proceedings of the VI International Conference on Sarcoidosis, edited by $\mathbf{K}$. Wai and Y. Hosoda, p. 253. University Park Press. 
Selvoos, O. and Niemisto, M. (1972). Tuberculin sensitivity in active and cured sarcoidosis in Finland. Proceedings of the VI International Conference on Sarcoidosis, edited by K. Wai and Y. Hosoda, p. 248. University Park Press.

Valentine, F. T. and Lawrence, H. S. (1969). Transfer factor. In Mediators of Cellular Immunity, edited by
H. S. Lawrence and M. Landy, p. 160. Academi $\frac{\text { Pे }}{4}$ Press, New York.

Requests for reprints to: Dr. W. L. Morison, Departmen of Dermatology, Massachusetts General Hospital, Boston Massachusetts, 02114, USA. 